\title{
PREVALÊNCIA DE INCONTINÊNCIA URINÁRIA EM ATLETAS PRATICANTES DE CORRIDA DE RUA
}

\author{
PREVALENCE OF URINARY INCONTINENCE IN PRACTICING ATHLETES OF RUNNING \\ Franciely da Rosa Ferraz ${ }^{a}$, Adriana Amaral Ferraz ${ }^{b}$, Erica Feio Carneiro Nunes ${ }^{c}$, \\ Gustavo Fernando Sutter Latorre ${ }^{\mathrm{d}}$ \\ ericacarneiro@uepa.bra , ericarneiro@yahoo.com.brb erica@perineo.net ${ }^{c}$, gustavo@perineo.net $^{\mathrm{d}}$
}

Data do recebimento do artigo: $21 / 04 / 2018$

Data do aceite: $02 / 07 / 2018$

\begin{abstract}
RESUMO:
Introdução: A incontinência urinaria (IU) é caracterizada pela perda involuntária da urina. Um dos fatores de risco para o desenvolvimento da IU são os exercícios de alta intensidade. Objetivo: Avaliar a prevalência de IU em atletas praticantes de corrida de rua. Metodologia: Estudo do tipo transversal, com amostra de 61 atletas corredores de rua de ambos os sexos. Foram aplicados 3 questionários: o International Consulation on Incontinence Questionnaire - Short Form (ICIQ-SF), King's health questionnaire (KHQ) e o protection, amount, frequency, adjustment, body (PRAFAB) em seus locais de treinamento. Resultados: 54,7\% eram mulheres, idade média de 36,38 $\pm 12,5$. A média de tempo de prática de atividade física foi de $99,2 \pm 110,7$ meses, os participantes praticavam $84,63 \pm 77,4$ meses a modalidade de corrida de rua, $67,9 \%$ corriam 42 $\mathrm{km}$. A prevalência de IU foi de 49,7\% em ambos os sexos. Grande severidade na sintomatologia da IU. Conclusão: prevalência em IU condizente com estudos anteriores, sendo o tipo de IU mais prevalente foi a IUE o que reforça a analise de demais autores. Por meio de nossos achados foi possível evidenciar que o não há um grande impacto na qualidade de vida dos atletas.
\end{abstract}

Palavras-chave: Incontinência urinária; fisioterapia; qualidade de vida; atletas.

\section{ABSTRACT:}

Introduction: Urinary incontinence (UI) is characterized by involuntary loss of urine. One of the risk factors for UI development is high intensity exercise. Objective: To evaluate the prevalence of UI in athletes practicing street racing. Methodology: A cross-sectional study with a sample of 61 street athletes of both sexes. Three questionnaires were applied: the International Consulation on Incontinence Questionnaire (ICIQ-SF), the King's health questionnaire (KHQ) and the protection, amount, frequency, adjustment, body (PRAFAB) at their training sites. Results: $54.7 \%$ were women, mean age of $36.38 \pm 12.5$. The mean time of practice of physical activity was $99.2 \pm 110.7$ months, the participants practiced $84.63 \pm 77.4$ months in the street racing mode, $67.9 \% \operatorname{ran} 42 \mathrm{~km}$. The prevalence of UI was $49.7 \%$ in both sexes. Great severity in UI symptoms. Conclusion: prevalence in UI consistent with previous studies, being the most prevalent UI type was SUI, which reinforces the analysis of other authors. Through our findings it was possible to show that there is not a great impact on the athletes' quality of life.

Keywords: Urinary incontinence; physical therapy; quality of life; athletes. 


\section{Introdução}

A incontinência urinária (IU) é um problema comum entre as mulheres, com consequente impacto negativo na qualidade de vida ${ }^{1,2}$. A IU é definida como a queixa de perda involuntária da urina ${ }^{3}$ e sua prevalência em jovens com idade de 15 a 44 anos na população geral varia de $6,2 \%$ a $12,4 \%$. Particularmente, a incontinência urinária por esforço (IUE) é definida como a queixa de perda involuntária da urina durante algum tipo de esforço como tosse e espirro. Trata-se da forma mais comum de IU feminina, cujas taxas de prevalência variam de $3 \%$ a $58,4 \%{ }^{6}$. As variações de prevalência podem ser explicadas pelos diferentes métodos utilizados e pelas populações estudadas ${ }^{7,8}$.

Há benefícios à saúde pela prática de exercícios físicos, inclusive algumas atividades físicas de moderada intensidade já foram demonstradas como efetivas na diminuição das chances de desenvolver IU, contudo o exercício físico intenso é listado como um dos principais fatores de risco para o surgimento do problema ${ }^{9,10}$, de forma que há indícios de que esportes de alto impacto podem resultar em alteração das pressões intra-abdominais refletidas aos órgãos pélvicos, induzindo a IUE durante o treino esportivo ou competições ${ }^{11,12}$. Tal fato pode se tornar constrangedor, afetando o desempenho esportivo ou até mesmo propiciando o abandono das atividades físicas ${ }^{13}$. Atletas que realizam atividades de alto impacto são as mais acometidas pela $\mathrm{IU}^{14}$. As atletas de elite, dançarinas ${ }^{15}$, de basquetebol e futebol de salão ${ }^{16}$ estão entre as mais atingidas.

A prática de exercícios está entre os elementos para uma vida saudável, bastante deseja atualmente, e assim, atividades como a corrida de rua ou o ciclismo se tornam corriqueiros na vida de milhares de brasileiros, porém são raros os estudos epidemiológicos sobre a IU em atletas praticantes de corrida de rua ${ }^{17,18}$, sendo este, por fim, o objetivo deste estudo.

\section{Metodologia}

O presente estudo teve caráter quantitativo com delineamento transversal. O respectivo projeto foi submetido e aprovado pelo Comitê de Éti- ca e Pesquisa da Faculdade Inspirar com parecer de liberação número 2364673.

Após entrar em contato com diversas equipes de corrida da cidade de Porto Alegre foi solicitado o e-mail dos atletas das equipes. Para os atletas que aceitaram participar do estudo foi marcado um horário para responderem os questionários nos locais de treinamento.

Foram incluídos atletas praticantes de corrida de rua maiores de 18 anos de ambos os sexos. Definiu-se como critério de exclusão atletas que foram submetidos a qualquer cirurgia prévia para correção de IU ou fecal e aqueles que faziam uso de diuréticos.

Os atletas que aceitaram responderam, inicialmente, questões sobre a modalidade de corrida que eles praticavam, se perdiam urina, o conhecimento a respeito do assoalho pélvico e se praticavam algum treinamento destes músculos. Em seguida foram entregues 3 questionários: o International Consulation on Incontinence Questionnaire - Short Form (ICIQ-SF), validado no Brasil, que avalia o impacto da IU para ambos os gêneros ${ }^{19,20}$. Seu escore geral é obtido pela soma dos escores das questões 3, 4 e 5. Quanto maior o escore maior o impacto sobre a qualidade de vida $^{21}$. O impacto sobre a qualidade de vida foi dividido de tal forma: nenhum impacto (0 ponto); impacto leve (de 1 a 3 pontos); impacto moderado (de 4 a 6 pontos); impacto grave (de 7 a 9 pontos); e impacto muito grave (10 ou mais pontos) ${ }^{22}$, o King's health questionnaire (KHQ) que se divide em 8 domínios relativos a qualidade de vida da pessoa com IU, o escore de qualidade de vida, que varia de 0 a 100 , considerando-se que quanto maior o número obtido, pior a qualidade de vida ${ }^{23}$ e por último o questionário protection, amount, frequency, adjustment, body (PRAFAB), que avalia a severidade da IU ainda não validado no Brasil, é composto por 5 questões sendo uma por tema (proteção, quantidade, frequência, adaptações e imagem corporal). Cada item pode ser pontuado de 1 a 4 , totalizando o mínimo de 5 e máximo de 20 pontos, quanto maior a pontuação, pior a severidade da $\mathrm{IU}^{24}$.

Os dados foram tabulados e a análise estatística foi realizada pelo programa SPSS versão 20. Os dados foram apresentados em médias e desvio padrão e percentagem da amostra. 


\section{Resultados}

Neste estudo foram incluídos 61 atletas corredores de rua, dos quais $43,5 \%$ eram homens com idade média de $38,38 \pm 10$ e $54,7 \%$ mulheres com idade média de $36,3 \pm 12$. A média de tempo de prática de atividade física foi de $99,2 \pm 110$ meses, os participantes praticavam $84,63 \pm 7$ meses a modalidade de corrida de rua.

Na Tabela 1 estão expressos em percentagem a distribuição dos dados acerca da IU e do assoalho pélvico. Importante ressaltar que $100 \%$ dos corredores que afirmaram perder urina eram mulheres.

Tabela 1: Distribuição dos dados acerca da IU e do assoalho pélvico da amostra.

\begin{tabular}{|c|c|c|c|c|}
\hline Variáveis & & $\%$ & & \\
\hline \multirow{2}{*}{$\begin{array}{l}\text { Qual a modalidade de cor- } \\
\text { rida }\end{array}$} & $5 \mathrm{~km}$ & $10 \mathrm{~km}$ & $21 \mathrm{~km}$ & $42 \mathrm{~km}$ \\
\hline & 32,4 & 47,2 & 13,2 & 7,2 \\
\hline \multirow{2}{*}{$\begin{array}{c}\text { Você já apresentou perda de } \\
\text { urina }\end{array}$} & Sim & Não & & \\
\hline & 32,1 & 67,9 & & \\
\hline \multirow{2}{*}{$\begin{array}{l}\text { Em que situação você perdeu } \\
\text { urina }\end{array}$} & Atividade física & Tosse ou espirro & Repouso & \\
\hline & 49,7 & 45,3 & 5 & \\
\hline $\begin{array}{l}\text { Há quanto tempo você nota } \\
\text { perda de urina }\end{array}$ & $\begin{array}{l}3 \text { meses } \\
12,5\end{array}$ & $\begin{array}{l}6 \text { meses } \\
37,5\end{array}$ & $\begin{array}{l}1 \text { ano } \\
25\end{array}$ & $\begin{array}{l}\text { Mais de } \\
1 \text { ano } \\
25\end{array}$ \\
\hline $\begin{array}{l}\text { Você já ouviu falar de assoa- } \\
\text { lho pélvico }\end{array}$ & $\begin{array}{l}\text { Sim } \\
45,3\end{array}$ & $\begin{array}{l}\text { Não } \\
54,7\end{array}$ & & \\
\hline $\begin{array}{c}\text { Realiza reforço de assoalho } \\
\text { pélvico }\end{array}$ & $\begin{array}{l}\text { Sim } \\
22,6\end{array}$ & $\begin{array}{l}\text { Não } \\
77,4\end{array}$ & & \\
\hline
\end{tabular}

Na Tabela 2 estão descritos a média e desvio padrão e percentagem dos resultados obtidos com o ICIQ- SF. 
Tabela 2: média e desvio padrão e percentagem dos resultados obtidos com o ICIQ- SF.

\begin{tabular}{|c|c|c|c|c|c|c|c|c|}
\hline Variáveis & \multicolumn{8}{|c|}{$\%$} \\
\hline $\begin{array}{l}\text { Frequência da perda } \\
\text { urina }\end{array}$ & $\begin{array}{c}\text { Nunca } \\
46,7\end{array}$ & $\begin{array}{c}\text { 1x por } \\
\text { semana ou } \\
\text { menos } \\
40\end{array}$ & $\begin{array}{r}2 \text { ou } 3 x \\
\text { por semana } \\
6,7\end{array}$ & $\begin{array}{l}1 \mathrm{x} \text { ao } \\
\text { dia } \\
0\end{array}$ & $\begin{array}{c}\text { Diver- } \\
\text { sas vezes } \\
\text { ao dia } \\
6,7\end{array}$ & $\begin{array}{r}\mathrm{O} \\
\text { tempo } \\
\text { todo } \\
0\end{array}$ & & \\
\hline $\begin{array}{l}\text { Quantidade de urina } \\
\text { que você pensa que perde }\end{array}$ & $\begin{array}{c}\text { Nenhuma } \\
69,4\end{array}$ & $\begin{array}{c}\text { pequena } \\
18,4\end{array}$ & $\begin{array}{l}\text { mode- } \\
\text { rada } \\
12,2\end{array}$ & $\begin{array}{l}\text { grande } \\
\quad 0\end{array}$ & & & & \\
\hline $\begin{array}{c}\text { Em geral quanto } \\
\text { perder urina interfere em } \\
\text { sua vida diária }\end{array}$ & $1,08 \pm 1,94$ & & & & & & & \\
\hline $\begin{array}{l}\text { Quando você perde } \\
\text { urina }\end{array}$ & $\begin{array}{c}\text { Nunca } \\
68\end{array}$ & $\begin{array}{r}\text { Perco } \\
\text { antes de } \\
\text { chegar ao } \\
\text { banheiro } \\
8\end{array}$ & $\begin{array}{l}\text { Perco } \\
\text { quando } \\
\text { tosse ou } \\
\text { espirro } \\
2\end{array}$ & $\begin{array}{l}\text { Perco } \\
\text { quando } \\
\text { estou dor- } \\
\text { mindo } \\
6\end{array}$ & $\begin{array}{c}\text { Perco } \\
\text { quando es- } \\
\text { tou fazendo } \\
\text { atividades } \\
\text { físicas } \\
2\end{array}$ & $\begin{array}{c}\text { Perco } \\
\text { quando } \\
\text { terminei } \\
\text { de urinar } \\
\text { e estou } \\
\text { me ves- } \\
\text { tindo } \\
8\end{array}$ & $\begin{array}{l}\text { Perco } \\
\text { sem razão } \\
\text { óbvia } \\
4\end{array}$ & $\begin{array}{l}\text { Perco o } \\
\text { tempo todo } \\
2\end{array}$ \\
\hline ICIQ-Score & & & & & & & & \\
\hline
\end{tabular}

A análise dos escores dos domínios do KHQ apresentou os valores correspondentes na Tabela 3.

Tabela 3: Média e desvio padrão (DP) por domínio do KHQ da amostra.

\begin{tabular}{cc}
\hline Domínio KHQ & Média \pm DP \\
\hline Geral de saúde & $14,63 \pm 26,14$ \\
Impacto & $20,54 \pm 24,26$ \\
LAD & $30,91 \pm 0,8$ \\
Limitações físicas & $31,08 \pm 8,3$ \\
Limitações sociais & $35,78 \pm 9,15$ \\
Relações Sociais & $30,61 \pm 1,42$ \\
Emoções & $33,08 \pm 8,9$ \\
Sono & $38,08 \pm 8,1$ \\
Gravidade & $35,08 \pm 8,91$ \\
\hline
\end{tabular}

$\mathrm{Na}$ avaliação da severidade pelo KHQ foram verificados os principais desconfortos dos atletas, conforme a Tabela 4. 
Tabela 4: Severidade dos sintomas urinários pelo KHQ.

\begin{tabular}{ccc}
\hline Sintomas & Indicador & \% \\
\hline Polaciúria & Um pouco & 68,9 \\
& Moderadamente & 18,6 \\
& Muito & 11,6 \\
Nocturia & Um pouco & 40 \\
& Moderadamente & 42 \\
& Muito & 17,8 \\
Urgência & Um pouco & 84,6 \\
& Moderadamente & 10,3 \\
Incontinência aos esforços & Muito & 5,1 \\
& Um pouco & 76,5 \\
& Moderadamente & 20,6 \\
Incontinência urinária-relação & Muito & 2,9 \\
sexual & Um pouco & 76,5 \\
& Moderadamente & 17,6 \\
& Muito & 5,9 \\
\hline
\end{tabular}

Os valores obtidos com o PRAFAB estão expostos na Tabela 5.

Tabela 5: Médias \pm DP e percentagem obtidas pelo PRAFAB.

\begin{tabular}{|c|c|c|c|c|c|}
\hline Variáveis & \multicolumn{5}{|c|}{$\%$} \\
\hline Proteção & $\begin{array}{c}\text { Nunca } \\
76,2\end{array}$ & $\begin{array}{c}\text { Às vezes } \\
11,9\end{array}$ & $\begin{array}{l}\text { Normalmente } \\
\text { uso protetores. } \\
4,8\end{array}$ & $\begin{array}{l}\text { Sempre uso } \\
\text { protetores } \\
7,1\end{array}$ & \\
\hline $\begin{array}{l}\text { Média do } \\
\text { Escore }\end{array}$ & \multicolumn{5}{|c|}{$1,42 \pm 0,9$} \\
\hline Frequência & $\begin{array}{c}\text { Perco vez por sema- } \\
\text { na ou sempre } \\
67,4\end{array}$ & $\begin{array}{c}\text { Perco entre } \\
1 \text { ou } 3 \text { vezes por } \\
\text { semana } \\
17,4\end{array}$ & $\begin{array}{c}\text { Mais de } 3 \text { vezes } \\
\text { por semana } \\
2,2\end{array}$ & $\begin{array}{l}\text { Perco todo } \\
\text { o dia } \\
\quad 4,3\end{array}$ & $\begin{array}{c}\text { Não se } \\
\text { aplica } \\
8,7\end{array}$ \\
\hline $\begin{array}{l}\text { Média do } \\
\text { Escore }\end{array}$ & \multicolumn{5}{|c|}{$1,7 \pm 1,27$} \\
\hline Quantidade & $\begin{array}{l}\text { Perco gotas ou } \\
\text { menos } \\
21,3\end{array}$ & $\begin{array}{c}\text { Às vezes perco } \\
\text { um jatinho } \\
17\end{array}$ & $\begin{array}{l}\text { Molha muito } \\
\text { meu protetor e } \\
\text { roupas } \\
4,3\end{array}$ & $\begin{array}{c}\text { Encharca } \\
\text { ou até vaza } \\
\text { meu protetor } \\
2,1\end{array}$ & $\begin{array}{c}\text { Não se } \\
\text { aplica } \\
55,3\end{array}$ \\
\hline $\begin{array}{l}\text { Média do } \\
\text { Escore }\end{array}$ & \multicolumn{5}{|c|}{$3,53 \pm 1,9$} \\
\hline Adaptações & $\begin{array}{l}\text { Não me prejudica } \\
\text { nas atividades diárias } \\
31,9\end{array}$ & $\begin{array}{l}\text { Parei com os } \\
\text { esportes e ativi- } \\
\text { dades } \\
8,5\end{array}$ & $\begin{array}{c}\text { Parei com a } \\
\text { maioria de minhas } \\
\text { atividades } \\
0\end{array}$ & $\begin{array}{l}\text { Praticamen- } \\
\text { te não saio de } \\
\text { casa } \\
0\end{array}$ & $\begin{array}{l}\text { Não se } \\
\text { aplica } \\
59,6\end{array}$ \\
\hline $\begin{array}{l}\text { Média do } \\
\text { Escore }\end{array}$ & \multicolumn{5}{|c|}{$3,5 \pm 1,9$} \\
\hline
\end{tabular}




\begin{tabular}{|c|c|c|c|c|c|}
\hline Autoimagem & $\begin{array}{l}\text { Minha incontinência } \\
\text { não me incomoda } \\
17,6\end{array}$ & $\begin{array}{c}\text { É irritante e } \\
\text { problemática, } \\
\text { mas não me } \\
\text { irrita muito } \\
8,8\end{array}$ & $\begin{array}{c}\text { Minha inconti- } \\
\text { nência me faz sentir } \\
\text { sujo } \\
8,8\end{array}$ & $\begin{array}{c}\text { Sinto nojo } \\
\text { de mim mesmo } \\
\text { por causa da } \\
\text { minha inconti- } \\
\text { nência } \\
0\end{array}$ & $\begin{array}{l}\text { Não se } \\
\text { aplica } \\
64,7\end{array}$ \\
\hline $\begin{array}{l}\text { Média do } \\
\text { Escore }\end{array}$ & \multicolumn{5}{|c|}{$3,85 \pm 1,65$} \\
\hline
\end{tabular}

\section{Discussão}

A corrida de rua é uma das modalidades esportivas mais praticadas no Brasil, podendo reunir mais de 25 mil pessoas em uma única prova ${ }^{25}$. $\mathrm{O}$ atletismo, a ginástica e o trampolim são considerados fatores de risco para o desenvolvimento de IU, pois são atividades de alto-impacto ${ }^{26,27}$, motivando o objetivo de estudo que foi verificar a prevalência de IU em corredores de rua.

Neste estudo observou-se que $32,1 \%$ dos praticantes de corrida de rua da amostra, todos do sexo feminino, apresentavam algum relato de "queixa" de perda de urina e na maioria esta perda ocorreu durante a atividade. Araújo et al. ${ }^{28}$ também encontrou alta prevalência de IU em corredoras de longa distância, ginastas e jogadoras de basquete, porém não relacionou a perda de urina com a capacidade de contração dos MAP, inferindo qu outros fatores devem estar envolvidos na etiopatogenia da IU em atletas.

A corrida, em especial a de longa distância causa fadiga muscular, que pode ocorrer nos MAP, visto que cerca de $70 \%$ de suas fibras musculares são do tipo I, ou seja, fibras de contração lenta, ricas em mitocôndrias, e que se contraem por mecanismo oxidativo ${ }^{29,30}$. Bo et al. ${ }^{31}$ relatam que o atleta de alto desempenho apresenta um ritmo de treinamento mais intenso que o atleta amador, o que faz com que os músculos do assoalho pélvico sejam expostos a sobrecargas e intensidades maiores. Recentemente, Matos et al. ${ }^{32} \mathrm{em}$ uma revisão sistemática concluiu que a prática esportiva aumenta a prevalência da IU e que o tipo de atividade desempenhada pelas mulheres também influencia o transtorno.

A maioria dos pesquisados revelou não conhecer o assoalho pélvico e nem exercita-lo, o que pode contribuir para sua disfunção. Neste sentido, Almeida et al. ${ }^{33}$ explicam que as mulheres envolvidas, em longo prazo, a esportes de alto impacto devem ser avisadas sobre o impacto dessas atividades nos MAP e desta forma, serem oferecidas estratégias preventivas de disfunções.

\section{Conclusão}

A prevalência de IU em corredores de rua foi alta, destacando-se que as mulheres são as mais atingidas. Há necessidade de estudo longitudinais que possam determinar o efeito da corrida nos MAP e na função urinária.

\section{Referências}

1. Hampel C, Artibani W, Espuña Pons M, Haab F, Jackson $\mathrm{S}$, Romero J, et al. Understanding the burden of stress urinary incontinence in Europe: A qualitative review of the literature. Vol. 46, European Urology. 2004. p. 15-27.

2. Chong EC, Khan AA, Anger JT. The financial burden of stress urinary incontinence among women in the United States. Curr Urol Rep. 2011;12(5):358-62.

3. Sultan AH, Monga A, Lee J, Emmanuel A, Norton C, Santoro $\mathrm{G}$, et al. An International Urogynecological Association (IUGA)/International Continence Society (ICS) joint report on the terminology for female anorectal dysfunction. Neurourol Urodyn. 2017;36(1):10-34.

4. Caylet N, Fabbro-Peray P, Marès P, Dauzat M, PratPradal D, Corcos J. Prevalence and occurrence of stress urinary incontinence in elite women athletes. Can J Urol. 2006;13(4):3174-9.

5. Al-Mukhtar Othman J, Åkervall S, Milsom I, Gyhagen M. Urinary incontinence in nulliparous women aged 2564 years: a national survey. In: American Journal of Obstetrics and Gynecology. 2017. p. 149.e1-149.e11.

6. Irwin D, Kopp Z, Agatep B, Milsom I, Abrams P. Worldwide prevalence estimates of lower urinary tract symptoms, overactive bladder, urinary incontinence and bladder outlet obstruction. BJU Int [Internet]. 2011;108(7):1132-9. Available from: http://www.ncbi. nlm.nih.gov/pubmed/21231991 
7. Bedretdinova D, Fritel X, Panjo H, Ringa V. Prevalence of Female Urinary Incontinence in the General Population According to Different Definitions and Study Designs. Eur Urol. 2016;69(2):256-64.

8. Minassian VA, Stewart WF, Wood GC. Urinary incontinence in women: Variation in prevalence estimates and risk factors. Obstet Gynecol. 2008;111(2):324-31.

9. Abrams P, Andersson KE, Birder L, Brubaker L, Cardozo $\mathrm{L}$, Chapple C, et al. Fourth international consultation on incontinence recommendations of the international scientific committee: Evaluation and treatment of urinary incontinence, pelvic organ prolapse, and fecal incontinence. Vol. 29, Neurourology and Urodynamics. 2010. p. 213-40.

10. Nygaard IE, Shaw JM. Physical activity and the pelvic floor. Vol. 214, American Journal of Obstetrics and Gynecology. 2016. p. 164-71.

11. Duarte G, Marcolin AC, Gonçalves CV, Quintana SM, Berezowski AT, Nogueira AA, et al. Infecção urinária na gravidez: análise dos métodos para diagnóstico e do tratamento. Rev Bras Ginecol e Obs [Internet]. 2002;24(7):471-7. Available from: http://www.scielo.br/scielo.php?script=sci_arttext\&pid=S0100-72032002000700007\&lng=pt\&n$\mathrm{rm}=\mathrm{iso} \& \mathrm{t} \operatorname{lng}=\mathrm{pt}$

12. Riss P, Kargl J. Quality of life and urinary incontinence in women. Maturitas. 2011;68(2):137-42.Knorst MR, Cavazzotto K, Henrique M, Resende TL. Interven????o fisioterap??utica em mulheres com incontin??ncia urin??ria associada ao prolapso de ??rg??o p??lvico. Brazilian J Phys Ther. 2012;16(2):102-7.

13. Papanicolaou S, Hunskaar S, Lose G, Sykes D. Assessment of bothersomeness and impact on quality of life of urinary incontinence in women in France, Germany, Spain and the UK. BJU Int. 2005;96(6):831-8.

14. Araujo Maíta Poli de, Parmigiano Tathiana R., Negra Laura Grechi Della, Torelli Luiza, Carvalho Camila Garcia de, Wo Liris et al. Avaliação do assoalho pélvico de atletas: existe relação com a incontinência urinária?. Rev Bras Med Esporte. 2015; 21(6): 442-446.

15. Bo K. Urinary incontinence, pelvic floor dysfunction, exercise and sport. Sports Med. 2004;34:451-64

16. Jácome C, Oliveira D, Marques A, Sá-Couto. Prevalence and impact of urinary incontinence among female athletes. Gynecology Obstetrics. 2011; 114(1): 60-63

17. Eliasson K, Edner A, Mattsson E. Urinary incontinence in very young and mostly nulliparous women with a history of regular organised high-impact trampoline training: Occurrence and risk factors. Int Urogynecol J. 2008;19(5):687-96.

18. Simeone C, Moroni A, Pettenò A, Antonelli A, Zani D, Orizio $\mathrm{C}$, et al. Occurrence rates and predictors of lower urinary tract symptoms and incontinence in female athletes. Urologia. 2010;77(2):139.
19. BERGHMANS, L. C. M. et al. Guia de Prática Clínica da Associação Brasileira de Fisioterapia Pélvica para Incontinência Urinária de Esforço. Brazilian Pelvic Physiotherapy Association Guideline on Stress Urinary Incontinence adapted from the Royal Dutch Society for Physiotherapy. Guia De Prática Clínica Da Abfp Rev. 2016.

20. TAMANINI, J. T. et al. Validation of the "International Consultation on Incontinence Questionnaire - Short Form" (ICIQ-SF) for Portuguese. Revista Saúde Pública, v. 38, n. 3, 2004. Disponível em: <http://repositorio.unicamp.br/bitstream/REPOSIP/17560/1/S003489102004000300015.pdf>. Acesso em: 06 abr 2017.

21. GOMES, P.L ; RIBEIRO, R.M. ; BARACAT, E.C. Tratamento não-cirúrgico da incontinência urinária de esforço: revisão sistemática. Revista Femina. V. 38, n. 7, São Paulo, 2010. Disponível em: <http://files.bvs.br/upload/S/0100-7254/2010/v38n7/a1520.pdf > acesso em: 30 de março de 2017

22. RICCETTO, C.; PALMA, P.; TARAZONA, A. Aplicaciones clínicas de la teoría integral de la continência. Actas Urol Esp, v. 29, n. 1, p. 31-40, 2005. Disponível em< http://scielo.isciii.es/pdf/aue/v29n1/revision.pdf $>$. Acesso em: 06 abr 2017.

23. FONSECA, E. S. M. et al. Validação do questionário de qualidade de vida (King's Health Questionnaire) em mulheres brasileiras com incontinência urinária Rev Bras Ginecol Obstet. v. 27, n. 5, p. 235-42, 2005. Disponível em<http://www.scielo.br/pdf/rbgo/v27n5/25638.pdf >Acesso em: 28 mar 2017.

24. Hendriks EJ, Bernards AT, Staal JB, et al (2008) Factorial validity and internal consistency of the PRAFAB questionnaire in women with stress urinary incontinence. BMC Urol8:1 .doi: 10.1186/1471-2490-8-1.

25. Evangelista AL.Treinamento de corrida de rua: uma abordagem fisiológica e metodológica. São Paulo: Phorte; 2010

26. Nygaard IE, Glowacki C, Saltzman CL. Relationship between foot flexibility and urinary incontinence in nulliparous varsity athletes. Obstet Gynecol . 1996;87(6):104951. [ Links ]

27. Bø K. Urinary incontinence, pelvic floor dysfunction, exercise and sport. Sports Med. 2004;34(7):451-64. [ Links ]

28. Araujo Maíta Poli de, Parmigiano Tathiana R., Negra Laura Grechi Della, Torelli Luiza, Carvalho Camila Garcia de, Wo Liris et al. Avaliação do assoalho pélvico de atletas: existe relação com a incontinência urinária? rev bras med esporte. 2015; 21(6): 442-446.

29. Bo K, Berghmans B, Morkved S, Van Kampen M. Evidence-based Physical Therapy for the Pelvic Floor: Bridging Science and Clinical Practice [Internet]. Vol. 27, Cardiovascular Imaging. 2007. 544 p. Available from: http:// www.lavoisier.fr/notice/fr283071.html 
30. Nygaard IE, Thompson FL, Svengalis SL, Albright JP. Urinary incontinence in elite nulliparous athletes. Obstet Gynecol. 1994;84(2):183-7.

31. Bø $\mathrm{K}^{1}$, Sundgot-Borgen J. Are former female elite athletes more likely to experience urinary incontinence later in life than non-athletes? Scand J Med Sci Sports. 2010 Feb;20(1):100-4. doi: 10.1111/j.1600-0838.2008.00871.x.

32. de Mattos Lourenco $\mathrm{TR}^{1,2}$, Matsuoka $\mathrm{PK}^{3}$, Baracat $\mathrm{EC}^{3}$, Haddad $\mathrm{JM}^{3}$. Urinary incontinence in female athletes: a systematic review. Int Urogynecol J. 2018 Mar 19. doi: 10.1007/s00192-018-3629-z. [Epub ahead of print]

33. Almeida MB, Barra AA, Saltiel F, Silva-Filho AL, Fonseca AM, Figueiredo EM. Urinary incontinence and other pelvic floor dysfunctions in female athletes in Brazil: A cross-sectional study. Scand J Med Sci Sports. 2016 Sep;26(9):1109-16. doi: 10.1111/sms.12546. Epub 2015 Sep 15.

\section{Como citar este artigo:}

Ferraz FR, Ferraz AA, Nunes AFC, Latorre GRF. Prevalência de incontinência urinária em atletas praticantes de corrida de rua . Rev. Aten. Saúde. 2018;16(57):37-44. 